\title{
Study of the areal density in the read heads with spin valves with nano-oxide-layer insertion
}

\author{
Daniela Ionescu ${ }^{1,}{ }^{*}$ and Gabriela Apreotesei ${ }^{2}$ \\ ${ }^{1}$ Gheorghe Asachi Technical University of Iasi, Department of Telecommunications, Carol I Blvd., \\ No.11, 700506 Iasi, Romania, \\ ${ }^{2}$ Gheorghe Asachi Technical University of Iasi, Department of Physics, Dimitrie Mangeron Blvd. 67, \\ 700050, Iasi, Romania
}

\begin{abstract}
Read heads performances have been analyzed by simulation (HFSS) in the case of the devices designed with a category of spin valve structures with nano-oxide-layer insertion, based on Co alloys. Different structures of the stack of layers in the spin valve were considered in order to increase the magnetic resistance due to contribution of the insertion layer. A parametrical evolution of the resistance-area product variation $\triangle R A$, and of the areal storage density have been obtained using the simulation and theoretical data. Results indicate areal densities up to $780 \mathrm{Gbit} / \mathrm{in}^{2}$ for the system manufactured in practice, when the variation of the resistance-area product was greater than $7.4 \ldots 9 \mathrm{~m} \Omega \bullet \mu \mathrm{m}^{2}$, strongly dependent on the nanooxide insertion layer. These performances can be overlapped by parameters correlation, indicated by simulation. Consequently, our simulations offer solutions for improving the recording performances by controlling the spin valve structure.
\end{abstract}

\section{Introduction}

The read/write heads of the hard disk, using spin valves with nano-oxide-layer insertion (NOL-SV), have the role to transfer the information (the succession of bits) between the magnetic storage media and the electronic components which processes the digital signal. The read heads are converting the magnetic pulses (bits) into voltage levels and have to be able to work with a high speed and a great sensitivity. The case of the perpendicular recording was considered, where the bits point up or down perpendicular to the disk surface, in comparison with the parallel recording case, where the bits are moving in the disk plane. Higher fields are necessary at perpendicular recording to set the magnetization, but the advantage is that the magnetization is more stable.

A category of NOL-SVs has been studied in this paper, for the advantages presented by such a kind of structures: an increased GMR ratio by NOL insertion inside the pinned layer and after the free layer, while the sense-layer can be implemented thinner than in the case of classical SV. The NOL-SV are used in giant magneto resistive (GMR) read heads, for magnetic recording disk drives where requirements for higher density recording have to be fulfilled. High magnetic resistance and thinner layers are necessary for spin valves and the

\footnotetext{
*Corresponding author: danaity@yahoo.com
} 
solution is represented by the specular reflection layers such as nano-oxide layers (NOLs) inserted in the spin valves.

\section{Microstructures with discontinuous oxidation layer}

\subsection{Theoretical considerations}

The NOL spin valves present a great magnetic resistance ratio (MR), which ensures a great sensitivity to the device used for applications [1], [2]. The theoretical model was based on the Valet-Fert theory [3], which represents the theory for giant magnetic resistance in the spin valves with current-perpendicular-to-plane (CPP) geometry. The theory applied in The NOLSV case is known as the modified Valet-Fert model for the current-perpendicular-to-plane giant magnetic resistance spin-valve with a current-confined-path nano-oxide layer. The current which is passed perpendicular to the ferro / nonferro-magnetic interfaces it is assumed that presents a uniform current density uniform across the area. The electronic transport in magnetic multilayers depends on the electrons scattering phenomena due to the spin-orbit interactions.

The specific resistance $A R$ has been computed in the case of the considered NOL-SV, like the product of the area $A$ through which a CPP current flows transverse on interfaces and the sample resistance $R$. The difference between the anti-parallel and parallel states (when the magnetizations of adjacent ferromagnetic layers in the stack are anti-parallel, respectively parallel) leads us to the specific resistance variation:

$$
\triangle A R=A R_{\text {antill }}-A R_{\mathrm{II}}
$$

Which represents the difference between the specific resistances in the anti-parallel and parallel states [2], [4].The CPP magnetic resistance ratio was computed as [5], [6]:

$$
M R_{\mathrm{CPP}}[\%]=\frac{\Delta A R}{A R_{\text {antill }}}
$$

The areal density at data storage was considered as the amount of data that can be stored in a given amount of hard disk platter and represents a measure of the number of bits that can be stored on the unit of area. Physically, the areal density can be calculated as the product of the track density and the linear or recording density. The track density indicates how many tracks can be placed down on an inch of radius on the platters. The linear or recording density indicates how tightly the bits are packed within a length of track (bits per inch per track, BPI). Every track on the surface of a platter has a different length, because they represent concentric circles, and different tracks are written with different densities. The maximum linear density used on each drive is indicated on datasheets.

\subsection{Characteristics of the structure}

In the case of the devices designed with a category of spin valve structures with nano-oxidelayer insertion, hard magnetic materials based on Co alloys have been considered $\left(\mathrm{CoCr}_{15} \mathrm{Pt}_{12}\right.$ - $\mathrm{CoCr}_{22} \mathrm{Pt}_{14}$, respectively CoCrTa). Different structures of the stack of layers in the spin valve were taken into account, in order to increase the magnetic resistance due to contribution of the insertion layers. 
The considered layers configuration in the spin valve read head is indicated in Figure 1. The sensing current flows perpendicular to the ferro / nonferro-magnetic interfaces, in the considered current-perpendicular-to-plane (CPP) geometry.

The magnetic resistance enhancement is due to the specular reflection effect inside the NOL-SV structure. The spin-polarized electrons scatter at the NOL surfaces, placed above the free layer and inside the pinned magnetic layer. The mean free path of majority spinpolarized electrons is consistently extended due to specular reflection at metal and insulator interfaces, on NOLs [7], [8], and [9]. In the same time, an improvement of the resistivity of the current-confined-path occurs [9], [10].

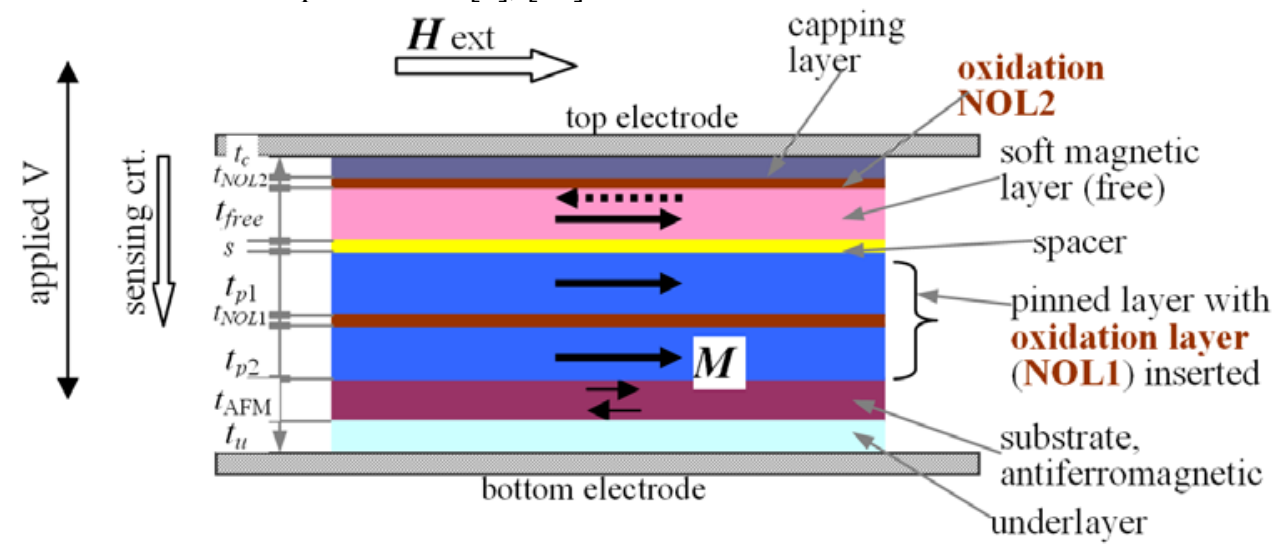

a)

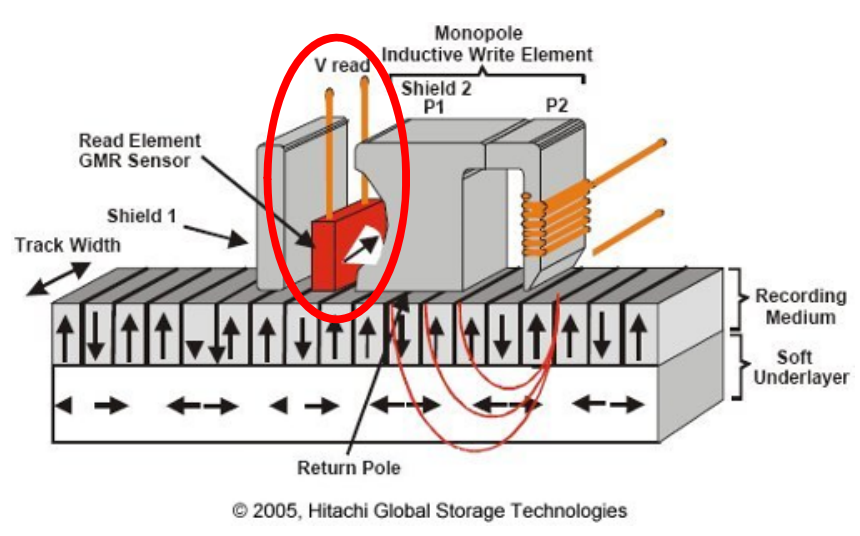

b)

Fig. 1. a) Layer configuration of the considered specular nano-oxide-layer spin valve. The currentperpendicular-to-plane (CPP) geometry was considered, where the sensing current flows perpendicular to the ferro / nonferro-magnetic interfaces. $b$ ) Read element with GMR sensor (inside the red oval), placed on a racetrack memory element, at perpendicular recording (after Hitachi Global Storage Technologies, 2005).

The NOL-SV structure of layers (from bottom to above layers) can be described: Ta (buffer, $3.7 \mathrm{~nm}$ ) / NiFeCr (2.4 nm) / PtMn (antiferromagnetic, $5.5 \mathrm{~nm}$ ) / CoCrPt (pinned, 1.8 $\mathrm{nm}$ ) // oxidation (NOL1) // CoCrPt (pinned, $2.2 \mathrm{~nm}$ ) / Ru (spacer, $2 \mathrm{~nm}$ ) / $\mathrm{CoCr}_{15} \mathrm{Pt}_{12}$ (free, $2.8 \mathrm{~nm}$ ) // oxidation (NOL2, $\mathrm{Ta}_{2} \mathrm{O}_{5}$ ) // Ta (capping layer, $3.5 \mathrm{~nm}$ ). Structure is placed on $\mathrm{Si}$ substrates.

The oxidation layers present a discontinuous structure, oxidation occurring preferentially along the grain boundaries on columnar directions as it was reported in literature [7], [11], 
and [12]. The discontinuous texture of the oxidation layers enhances the specular scattering and, in the same time, maintains the strong direct exchange coupling between the PtMn pinning layer and the pinned hard magnetic material, increasing the GMR effect.

\section{Results for the areal density}

The performances of the read heads with NOL-SV have been analyzed by simulation, using the HFSS program 13.0. A parametrical evolution of the resistance-area product variation $\triangle R A$, and of the areal storage density have been obtained, using the simulation and theoretical data.

In Figure 2 was represented the areal density of hard disk drives in function of the CPP magnetic resistance ratio of the considered spin valves. The external magnetic field and the sensing current for the spin valve have been indicated. The thicknesses of the first oxidation layer NOL1 place inside the pinned layer, $t_{\mathrm{NOL} 1}$, respectively the thickness of the pinned layer region situated above NOL1, $t_{p 1}$, were considered as parameters and the influence of their modification on the results was illustrated on graphs.

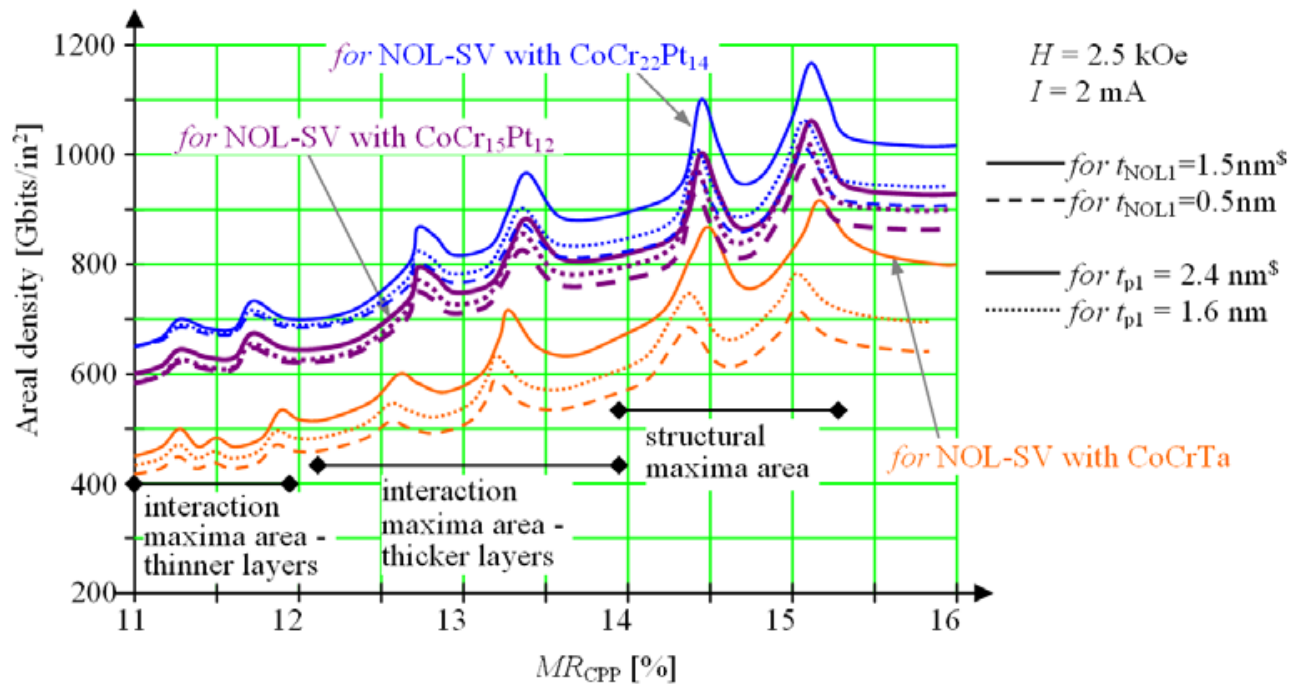

Fig. 2. Areal density of hard disk drives in function of the CPP magnetic resistance ratio of the considered spin valves. Structural, respectively interaction maxima were illustrated on graph. Internal parameters are correlated in order to obtain optimal results (correlated values are marked with \$).

The presence of groups of single and multiple maxima can be noticed on graphs.

The main structural maxima of the areal density are imposed by the magnetic ion in the hard magnetic alloy and their characteristics depend on the internal interactions in the exchange coupled system, hard - soft magnetic. A few interaction maxima, generally less intense, are controlled by other factors, like the resonant coupling phenomena between structure, external field and sensing current.

Two zones can be remarked in the interaction maxima area, namely an area with maxima obtained for thicker magnetic layers (3 - $4 \mathrm{~nm})$, respectively for thinner magnetic layers (2 $3 \mathrm{~nm}$ ), for which the magnetization is lower.

When different parameters of the structure have been modified, maxima with magnitude depending on the spin current were identified. Their originate in the magnetization dynamics, which is determined by the torques moving the spins, torques generated by the exchange interaction between conduction electrons and DW magnetizations, under the influence of external magnetic field. 
The areal storage density modifications in function of the specific resistance variations were calculated and represented on graphs in Figure 3. Graphs are useful for the practical read heads design and setting the material manufacturing criteria.

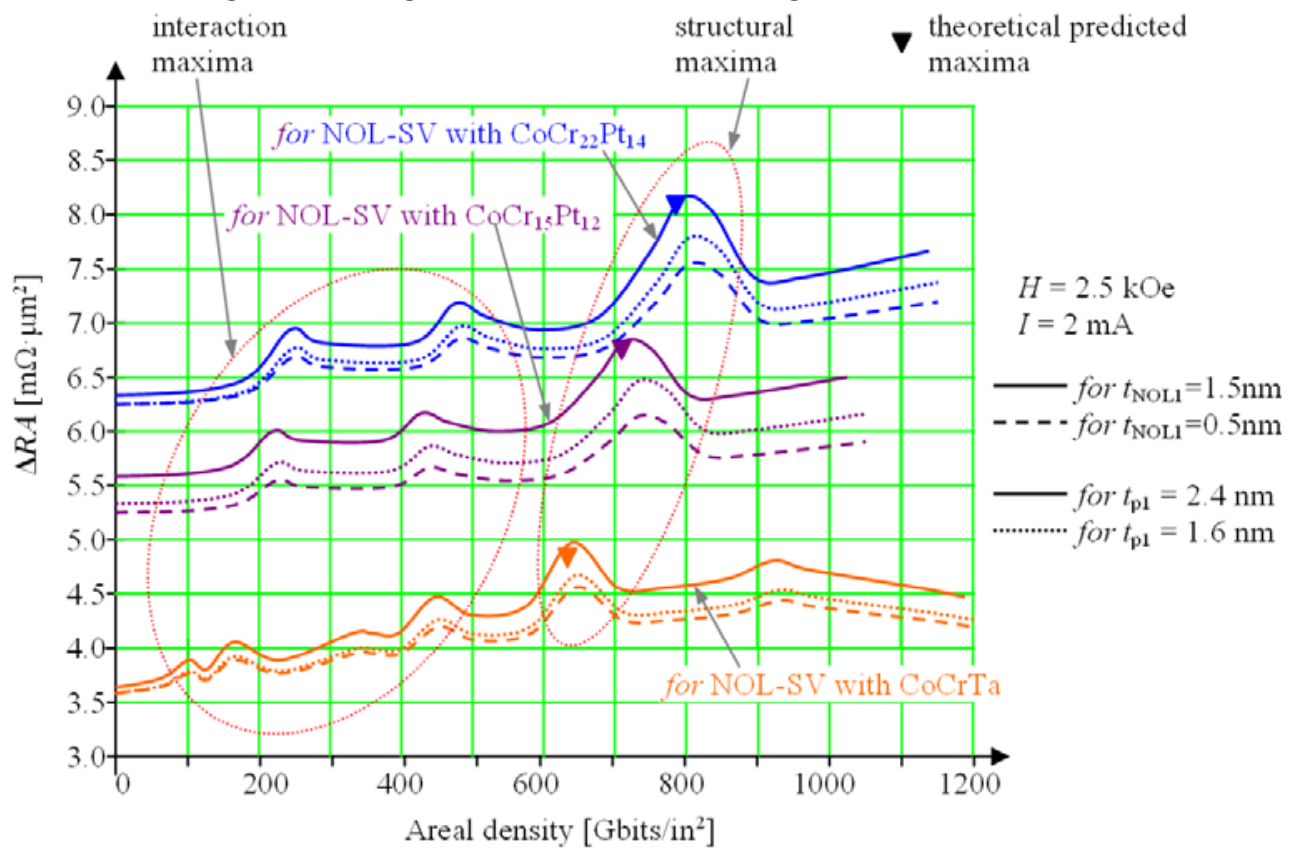

Fig. 3. Evolution of the areal density for a continuous modification of the specific resistance variation, for the considered spin valves. Structural, respectively interaction maxima of the $\triangle R A$, obtained for different subdomains of the areal density values, are indicated on graphs. Internal parameters are not entirely correlated in order to obtain optimal results.

One can remark maxima on the areal density graphs, for different subdomains of the areal density values. These maxima can be characterized like structural and interaction maxima, in function of physical mechanism which generates them.

The main structural maxima of the specific resistance variation, which corresponds to the higher values area for areal density, are imposed again by the magnetic ion nature in the hard magnetic alloy. The maximum magnitude depends on the exchange interactions strength. If the alloy structure changes, the maximum shifts consistently and also its magnitude modifies.

For each type of magnetic material, interaction maxima occurs, less intense than the structural maximum. These maxima are present for materials with a common magnetic ion (Co in our case), and are controlled by the resonant phenomena occurring at structure level when structure interacts with external excitation. The magnitude of these maxima depends mostly on the internal structure of the alloy.

Internal parameters are not entirely correlated in order to obtain optimal results.

Superior performances of the spin valve read heads can be achieved by correlation of the magnetic material nature and geometrical parameters of the magnetic layers, spacer and oxidation layers in the spin valve, as simulations indicate.

\section{Conclusions}

Results indicate maximal areal densities up to $780 \mathrm{Gbit} / \mathrm{in}^{2}$, when the variation of the resistance-area product was greater than $7.4 \ldots 9 \mathrm{~m} \Omega \cdot \mu \mathrm{m}^{2}$, strongly dependent on the nanooxide insertion layer. These represent the usually reached values for the SV systems 
manufactured in practice [1], [9], [13]. These values are lower that the maximal values indicated by the areal density - MR curves obtained by simulation with parameters correlation. On the latest mentioned curves, superior values for areal density (over 800 - 1000 Gbit $/ \mathrm{in}^{2}$ ) can be reached only in systems where the magnetic resistance ratio overlaps a threshold of about $14 \%$. An extremely careful correlation of all physical and geometrical parameters of the SV has to be practiced in order to obtain these results. Simulations can indicate the values of the necessary parameters which can ensure such a level of performance, in a non-invasive way.

Consequently, the main advantages of the analyzed NOL-SV structures can be pointed out as: higher MR ratio at not very high values of $R A$, corresponding to areal density maxima in the read heads.

Our simulations offer solutions for improving the recording performances by controlling the NOL spin valve structure.

\section{References}

1. C. Nam, Y. Jang, K. S. Lee, J. Shim, B. K. Cho, J. Nanosci Nanotechnol. 6, 11 (2006)

2. J. O. Ventura, J. B. Sousa, P. P. Freitas, A. Veloso, Journal of Magnetism and Magnetic Materials 272-276 (2004)

3. T. Valet, A. Fert, Phys. Rev. B 48, 7099 (1993)

4. L. Chen, Microsc Microanal 11(Suppl 2) (2005)

5. A. Gupta, S. Mohanan, M. Kinyanjui, A. Chuvilin, U. Kaiser, U. Herr, Journal of Applied Physics 107, 093910 (2010)

6. Teruya Shinjo (Ed.), Nanomagnetism and Spintronics, Second Edition (Elsevier, London UK, 2013)

7. D. H. Lee, S.Y. Yoon, J. H. Kim, S. J. Suh, Asian-European international conference on plasma surface engineering; Jeju City, Republic of Korea, 28 Sep. - 3 Oct. 2003, S00406090(04)00956-3 (2003)

8. H. D. Quang, N. T. Hien, S. K. Oh, N. H. Sinh, S. C. Yu, Journal of Physics D: Applied Physics 37, 23 (2004)

9. Hideaki Fukuzawa, Hiromi Yuasa and Hitoshi Iwasaki, Journal of Physics D: Applied Physics 40, 5 (2007)

10. Katsuaki Sato, Eiji Saitoh (Eds.), Spintronics for Next Generation Innovative Devices (John Wiley \& Sons, UK, 2015)

11. F. Shen, Q. Y. Xu, G. H. Yu, W. Y. Lai, Z. Zhang, Z. Q. Lu, G. Pan, A. Al-Jibouri, Applied Physics Letters 80, 23 (2002)

12. Y. Xu, D. D. Awschalom, J. Nitta, Junsaku (Eds.), Handbook of Spintronics (Springer Netherlands, 2016)

13. D. Weller, L. Folks, W. Lee, J.-U. Thiele, IEEE Transactions on Magnetics 36, 1 (2000) 